\title{
ARTICLE
}

\section{Activation of $\beta$-catenin signaling in aggrecan-expressing cells in temporomandibular joint causes osteoarthritis-like defects}

\author{
Tianqian Hui ${ }^{1,2,3}$, Yachuan Zhou ${ }^{3}$, Tingyu Wang ${ }^{4}$, Jun $\mathrm{Li}^{3,5}$, Shanxing Zhang ${ }^{3}$, Lifan Liao ${ }^{3}$, Jianhong $\mathrm{Gu}^{3}$, Ling Ye ${ }^{2}$, Lan $\mathrm{Zhao}^{3}$ and \\ Di Chen ${ }^{3}$
}

$\beta$-Catenin plays a critical role in cartilage formation and development. To further understand the role of $\beta$-catenin in osteoarthritis (OA) development in temporomandibular joint (TMJ), we have generated $\beta$-catenin conditional activation mice $\left(\beta\right.$-cat(ex3) $\left.{ }^{A g c 1 C r e E R}\right)$ by breeding Agc1-CreER mice with $\beta$-catenin flox(ex3)/+ mice. Results of histologic analysis showed the progressive TMJ defects in 3and 6-month-old $\beta$-cat (ex3) ${ }^{\text {Agc1CreER }}$ mice (tamoxifen induction was performed at 2 weeks of age), including decreased chondrocyte numbers in the superficial layer associated with less Alcian blue staining, increased numbers of hypertrophic chondrocytes in deep layers, and rough articular surface. Compared to the TMJ phenotype of $\beta$-cat(ex3) ${ }^{\text {Col2CreER }}$ mice, $\beta$-cat(ex3) ${ }^{\text {Agc1CreER }}$ mice showed much severe morphological defects in the superficial layer of TMJ. This may reflect that Agc1-CreER mice could efficiently target cells in the superficial layer of TMJ. Results of immunostaining showed significantly increased expression of MMP13, Col-X, Adamts4, and Adamts5 in TMJ of $\beta$-cat(ex3) ${ }^{\text {Agc1CreER }}$ mice. Results of proliferating cell nuclear antigen (PCNA), Ki67, and terminal deoxinucleotidyl transferase-mediated dUTP-fluorescein nick end labeling (TUNEL) staining further demonstrated that cell proliferation was decreased and cell apoptosis was increased in condylar cartilage of $\beta$-cat(ex3) ${ }^{\text {Agc } 1 \text { CreER }}$ mice. Our findings indicate that abnormal upregulation of $\beta$-catenin in TMJ leads to defects assembling to OA-like phenotype, further demonstrating that $\beta$ catenin plays a critical role in TMJ pathogenesis.

International Journal of Oral Science (2018)10:13 https://doi.org/10.1038/s41368-018-0016-z

\section{INTRODUCTION}

The temporomandibular joint (TMJ) is one of the most common sites affected by osteoarthritis (OA). It has been reported that up to 10 million Americans suffer from TMJ disorders (TMDs) each year and $14.56 \%$ of mainland Chinese patients with TMD had radiographic signs of $O A .^{1,2}$ Among TMDs, OA is the most prevalent degenerative disease. ${ }^{3}$ TMJ OA is characterized by cartilage degradation, alterations of subchondral bone remodeling, chronic pain, and joint dysfunction. ${ }^{4,5}$ Although TMJ OA is a common degenerative joint disease that affects TMJ cartilage during the aging process, the pathological mechanisms of this disease remain largely unknown. ${ }^{6}$

Canonical $\mathrm{Wnt} / \beta$-catenin signaling plays an important role in the development and progression in multiple forms of arthritis, such as $\mathrm{OA}^{7}$ spondyloarthritis, ${ }^{8-10}$ and diffuse idiopathic skeletal hyperostosis. ${ }^{11-13}$ It has been shown that conditional activation of $\beta$-catenin in knee joint cartilage and intervertebral disc cartilage leads to knee OA and disc tissue degeneration. ${ }^{7,14}$ In most recent studies, we also found that activation of $\beta$-catenin signaling in facet joint also causes severe OA-like phenotype (unpublished data). Our goal is to have comprehensive understanding of the role of $W n t / \beta$-catenin signaling in the pathogenesis of arthritis.

TMJ OA is one of the important forms of OA and is a common dental disease. The pathological progression of TMJ OA is considered to be a similar disease as knee OA. ${ }^{15}$ In previous studies, we generated $\beta$-cat $(e \times 3)^{\operatorname{Col} 2 \text { CreER }}$ mouse model and demonstrated that dysregulation of $\beta$-catenin causes OA-like cartilage degeneration in the TMJ tissue. ${ }^{16}$ We suggest that $\beta$ catenin is a critical molecule in OA pathogenesis. Interestingly, there is no significant change in the superficial zone of TMJ in $\beta$ cat $(e \times 3)^{\operatorname{Col} 2 \text { CreER }}$ mice. And cell proliferation and apoptosis was not changed upon $\beta$-catenin activation in this mouse model. ${ }^{16}$ TMJ condylar cartilage is comprised of dense extracellular collagen fibers and proteoglycans. ${ }^{17}$ The condylar cartilage is divided into the superficial, middle, and deep layers. ${ }^{18}$ The superficial and/or middle zones of condylar cartilage have been identified as regions enriched with highly proliferative cells. ${ }^{19}$ Mandibular condylar chondrocyte apoptosis and extracellular matrix degradation play an important role in the development of cartilage degeneration in TMJ OA. ${ }^{20,21}$ Moreover, activation of chondrocyte hypertrophy with low metabolism followed by apoptosis in the condylar cartilage is also considered to be part of the disease pathology associated with condylar cartilage degeneration. ${ }^{22}$ We propose that the $\beta$-cat $(e x 3)^{\text {Col2CreER }}$ mice might not be able to fully reveal the pathogenesis of TMJ OA.

We have recently examined the targeting specificity and recombination efficiency of $A g c 1-C r e E R^{T 2}$ mice in TMJ tissue and found that $A g c 1-C r e E R^{T 2}$ mice could efficiently target entire

\footnotetext{
${ }^{1}$ Department of Pediatric Dentistry, Peking University School and Hospital of Stomatology, Beijing, China; ${ }^{2}$ State Key Laboratory of Oral Diseases \& National Clinical Research Center for Oral Diseases \& West China Hospital of Stomatology, Sichuan University, Chengdu, China; ${ }^{3}$ Department of Orthopaedic Surgery, Rush University Medical Center,

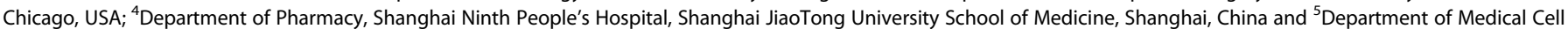
Biology and Genetics, Shenzhen Key Laboratory and the Center for Anti-Ageing and Regenerative Medicine, Shenzhen University Medical School, Shenzhen, China Correspondence: Di Chen (di_chen@rush.edu)
}

Accepted: 6 February 2018

Published online: 23 April 2018 
condylar cartilage, including superficial, middle, and deep layers. We decided to use this mouse model to re-evaluate the functions of $\beta$-catenin in TMJ tissue using the new $\beta$-cat $(\text { ex } 3)^{\text {Agc1ER }}$ conditional activation mouse model. It has been suggested that mechanisms of the aggrecan- or collagen-induced arthritis are very different. ${ }^{23}$ This may be related to the difference of their expression patterns in the condylar cartilage. Another advantage of using Agc1-CreER ${ }^{T 2}$ mice is that these mice could target cartilage tissue in adult animals. ${ }^{24}$ In the present study, we have used Agc1-CreER ${ }^{T 2}$ mice to drive $\beta$-catenin overexpression and determined the pathogenesis caused by $\beta$-catenin activation in the TMJ tissue. In our study, we explored whether overexpression of $\beta$-catenin in aggrecan-expressing chondrocytes could lead to cartilage matrix degradation and affect cell proliferation and apoptosis, which may contribute to the OA phenotype observed in $\beta$-cat $(\text { ex3 })^{A g c 1 C r e E R}$ mice.

\section{RESULTS}

High Cre-recombination efficiency and $\beta$-catenin activation in $\beta$ cat(ex3) ${ }^{\text {Agc1 CreER }}$ mice

To evaluate the Agc1-Cre expression and recombination efficiency in the TMJ cartilage, Agc1-CreER ${ }^{T 2}$ mice were bred with $R O S A^{m T / m G}$ reporter mice to generate $A g c 1-C r e E R^{T 2} ; R O S A^{m T / m G}$ mice. Tamoxifen was administered when the mice were aged 2 weeks and TMJ samples were harvested at 1 month. The red fluorescent image of condylar cartilage revealed no recombination in Cre-negative control mice (Fig. 1a). The green-labeled chondrocytes in Agc1$\mathrm{CreER}^{T 2}$; ROSA $\mathrm{m}^{\mathrm{T} / \mathrm{mG}}$ mice showed Agc1-expressing cells in the superficial, middle, and deep layers of condylar chondrocytes (Fig. 1a). We then generated $\beta$-cat (ex3) $)^{A g c 1 C r e E R}$ mice by crossing Agc1-CreER $R^{T 2}$ mice with $\beta$-catenin $(\text { ex } 3)^{\text {flox } / \text { flox }}$ mice. Tamoxifen was administered to 2-week-old mice and condylar cartilage samples were harvested from these mice at 3 and 6 months of age. Immunohistochemical $(\mathrm{IHC})$ results showed that $\beta$-catenin was overexpressed in the majority of condylar chondrocytes at 3-and 6 -month-old mice (Fig. 1b, c). There were few $\beta$-catenin-positive cells in the chondrocytes of $\mathrm{Cre}^{-}$mice. However, in $\beta$-cat $(\text { ex3 })^{\text {Agc1CreER }}$ mice, $\beta$-catenin expressed in the superficial, middle, and deep layers of condylar chondrocytes indicating that $\beta$-catenin in the chondrocytes was significantly increased compared to the $\mathrm{Cre}^{-}$mice (Fig. 1b, c). The numbers of $\beta$-catenin-positive cells in TMJ cartilage were significantly higher in $\beta$-cat $(\text { ex } 3)^{A g c 1 C r e E R}$ mice compared to $\mathrm{Cre}^{-}$mice (Fig. 1d). These results demonstrated that Agc1-CreER ${ }^{T 2}$ mice could target the chondrocytes of TMJ with high efficiency and drive $\beta$-catenin activation in condylar chondrocytes.

Conditional activation of $\beta$-catenin induced condylar cartilage defects

The role of $\beta$-catenin in condylar cartilage was investigated in $\beta$ cat $(e x 3)^{A g c 1 C r e E R}$ mice. Tamoxifen was administered to 2 -week-old mice and TMJ samples were harvested from these mice at 3 and 6 months of age. The chondrocytes in the control mice were well organized in the three layers: small and round cells in the top superficial layer; medium-sized cells were present in large numbers in the middle layer; and fewer, bigger, hypertrophic, mature cells in the deep layer (Fig. 2a, b, left panels). In contrast, 3month-old $\beta$-cat(ex 3$)^{A g c 1 C r e E R}$ mice presented early signs of TMJ $O A$ : decreased chondrocyte numbers in the superficial and middle layer accompanied with less Alcian blue staining in these areas, rough articular surface with numerous rounded chondrocytes often appearing as doublets, and cells in the middle and deep layers illustrated increased numbers of hypertrophic cells. In addition to decreased cellularity of the middle layers of cartilage, clustering of hypertrophic chondrocytes appeared more frequently in the deeper layer; cartilage area scattering and subchondral bone sclerosis were also observed in the $\beta$-cat $(e \times 3)^{A g c 1 C r e E R}$ mice compared with age-matched control group
(Fig. 2a, right panel). At 6 months of age, increased severity of defects, such as clustering chondrocytes in the superficial and deeper layer, the increasing numbers of hypertrophic chondrocytes, and subchondral new bone formation in condylar cartilage

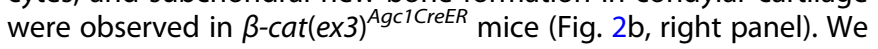
also analyzed the histology sections using the scoring system recommended by the Osteoarthritis Research Society International (OARSI) as previously described. ${ }^{27,29}$ We found that $\beta$-cat $(\text { ex3 })^{A g C 1 C r e E R}$ mice had significantly higher scores for OA damage compared to $\mathrm{Cre}^{-}$mice (Fig. 2c). The histomorphometric analysis also showed significant reductions in articular cartilage area in 3and 6-month-old $\beta$-cat(ex3) $)^{A g c 1 C r e E R}$ mice (Fig. 2d).

Changes in the expression of genes encoding for matrixdegradation enzymes in $\beta$-cat(ex3) ${ }^{\text {Agc IER }}$ mice

We have previously observed significant upregulation of Mmp13 and Adamts 5 expression in $\beta$-cat (ex3) ${ }^{\text {Col2CreER }}$ mice and demonstrated that both matrix metalloproteinase 13 (MMP13) and Adamts5 play important roles in the TMJ OA development in these mice. ${ }^{16}$ We propose that Mmp13 and Adamts5 might be the key downstream target genes of $\beta$-catenin during TMJ OA development. To further test this hypothesis, we performed IHC and immunofluorescence (IF) assays to determine changes in the expression of these collagenase and aggrecanases. Results of IHC revealed increased MMP13 expression in the $\beta$-cat $(e x 3)^{A g c i C r e E R}$ mice, especially in the superficial layer and deeper layer of the condylar cartilage in 3- and 6- monthold mice compared to controls (Fig. 3a, b). In addition, IF results showed significant increased ColX expression in chondrocytes of entire condylar cartilage in $\beta$-cat(ex3) $)^{A g c 1 C r e E R}$ mice compared to controls, indicating that the chondrocytes underwent hypertrophy at this stage (Fig. 4a, b). Furthermore, the expression of cartilagedegrading enzymes, such as Adamts4, and Adamts5 was also increased, especially in the superficial layer of TMJ chondrocytes in $\beta$ cat(ex3) Agc1CreER mice (Fig. 4c-f). These results suggest that the activation of $\beta$-catenin signaling could lead to chondrocyte hypertrophy and degenerative defects.

Alterations of cell proliferation and apoptosis in $\beta$-cat (ex3) $)^{\text {Agc1CreER }}$ mice

To further investigate the pathological process in $\beta$ - cat $(e \times 3)^{A g C 1 C r e E R}$ mice, proliferating cell nuclear antigen (PCNA), Ki67, and terminal deoxinucleotidyl transferase-mediated dUTP-fluorescein nick end labeling (TUNEL) staining was performed to assess changes in chondrocyte proliferation and apoptosis. In control mice, especially at the 3-month-old, results of PCNA staining showed that abundant proliferating cells were present in the entire TMJ cartilage. However, the PCNA-positive cells were dramatically reduced in the condylar cartilage of 3- and 6month-old $\beta$-cat(ex3) Agc1CreER mice (Fig. 5a). To further analyze changes in cell proliferation, we also performed Ki67 staining and found that numbers of Ki67-positive cells in the middle zones of condylar cartilage were significantly reduced in 3-month-old $\beta$-cat $(e \times 3)^{\text {Agc1CreER }}$ mice (Fig. 5b, c). These results suggest that overexpression of $\beta$-catenin in aggrecan-expressing condylar chondrocytes significantly affects cell proliferation. Data of TUNEL staining demonstrated the increased apoptotic cells, mostly in the deeper layers of the condylar cartilage of the mutant mice compared to that in control mice (Fig. 5d, e). In the control group, only few scattered apoptotic cells were detected in the deeper layer of the condylar cartilage in the mice at 6 months of age. Taking together, these results indicate that conditional activation of $\beta$-catenin in the TMJ tissue induced degenerative defects that might be partly due to changes in cell proliferation and apoptosis.

\section{DISCUSSION}

In this study, we generated $\beta$-cat(ex3) ${ }^{\text {Agc1CreER }}$ mouse model and demonstrated that overexpression of $\beta$-catenin in aggrecan- 
a
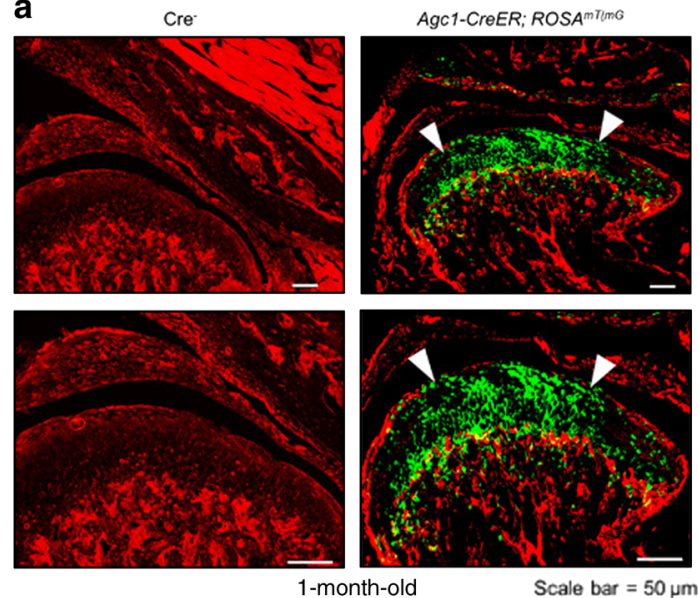

b
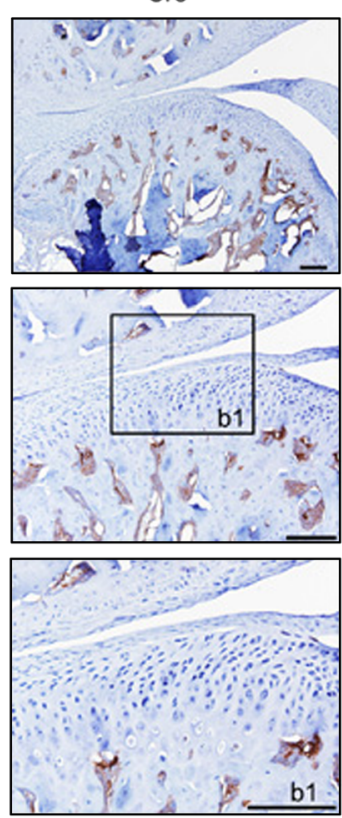

3-month-old
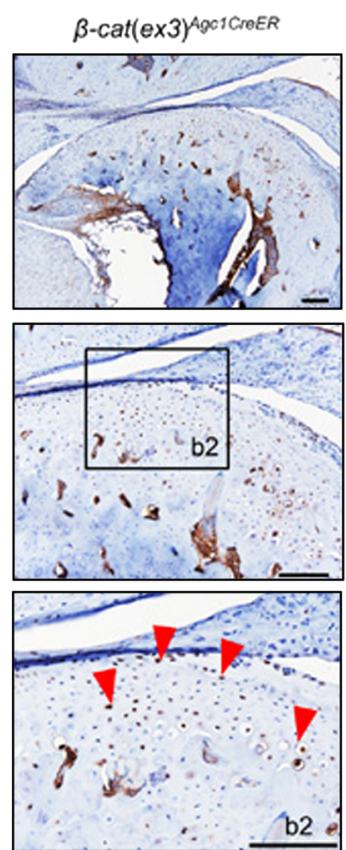

Scale bar $=50 \mu \mathrm{m}$
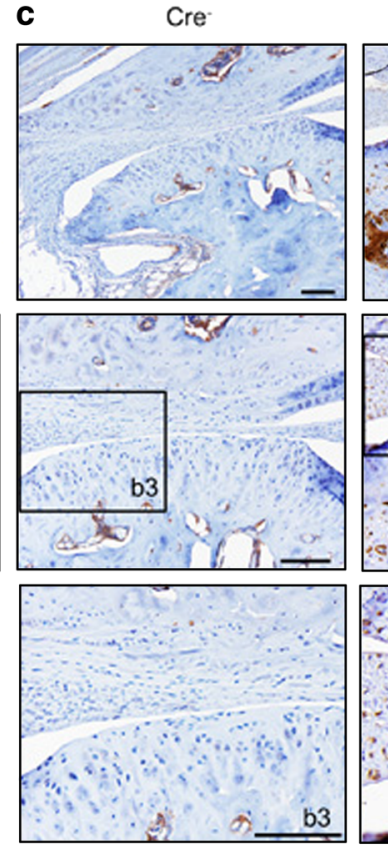

6-month-old $\beta$-cat(ex3) AgctcroER
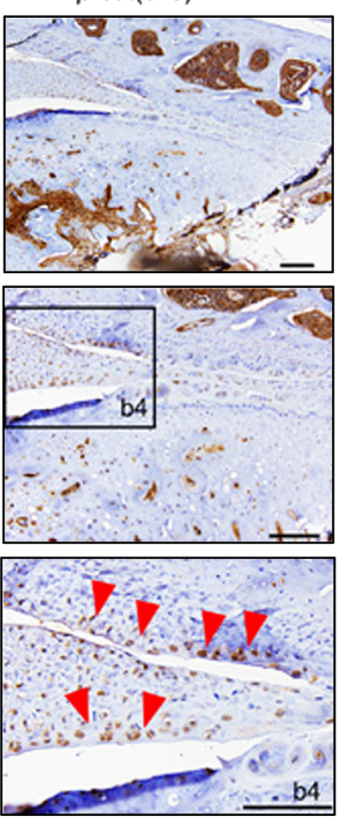

Scale bar $=50 \mu \mathrm{m}$

d

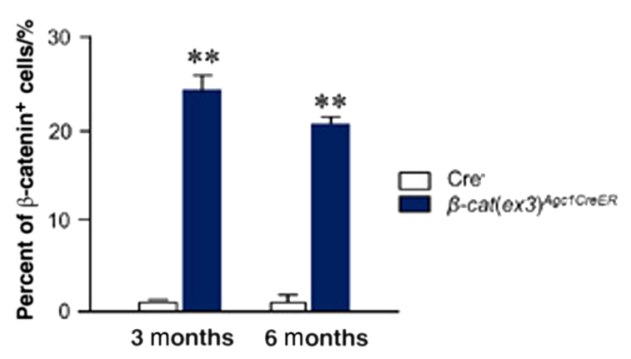

Fig. 1 Agc1-CreER directed Cre recombination in temporomandibular joint (TMJ) chondrocytes. a $A g c 1-C r e E R^{T 2} ; R O S A^{m T / m G}$ mice were generated by breeding Agc1-CreER ${ }^{T 2}$ transgenic mice with $R O S A^{m T / m G}$ reporter mice. TMJ samples were harvested from 1-month-old mice after they were injected with tamoxifen at the age of 2 weeks for 5 consecutive days. Histologic sections from $R O S A^{m T / m G}$ mice (Cre $\left.{ }^{-}\right)$and $A g c 1-$ $C r e E R^{T 2}$; ROSA ${ }^{m T / m G}$ mice were analyzed using fluorescence microscopy. High efficiency of Cre recombination (white arrowheads: Agc1-CreER ${ }^{T 2}$ targeting cells) in the TMJ chondrocytes, including the superficial, middle, and deep layers of condylar chondrocytes was found in Agc1$C r e E R^{T 2} ; R O S A^{m T / m G}$ mice. b, c Immunohistochemical (IHC) analysis showed that $\beta$-catenin expression was significantly increased in chondrocytes of 3-and 6-month-old $\beta$-cat (ex3) ${ }^{A g c 1 C r e E R}$ mice. Red arrowheads indicate $\beta$-catenin-positive chondrocytes. $\mathbf{d}$ Quantitative analyses of $\beta$-catenin-positive chondrocytes. A significant increase in the numbers of $\beta$-catenin-positive cells was observed in $\beta$-cat(ex3) ${ }^{A g c 1 C r e E R}$ mice compared to $\mathrm{Cre}^{-}$mice ${ }^{* *} P<0.01$; values are expressed as mean \pm standard erros; $n=5$ per group) 
a
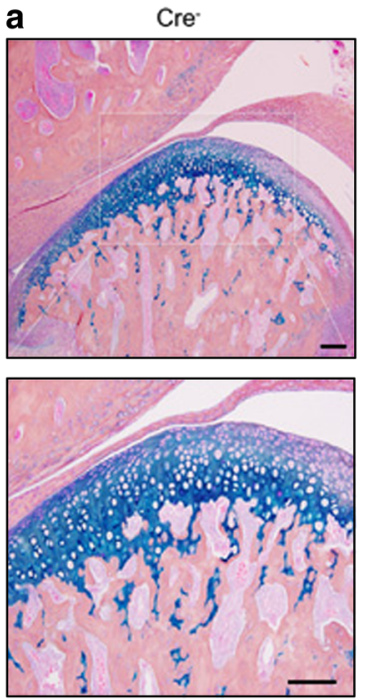

3-month-old $\beta-\operatorname{cat}(e \times 3)^{A g c t C r e E R}$
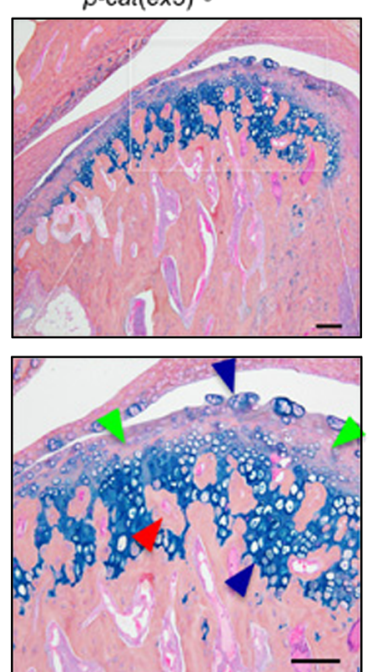

b
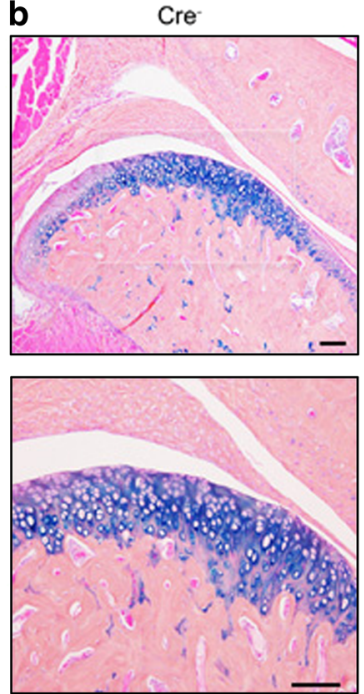

$\beta-c a t(e \times 3)^{A g c t c r o E R}$
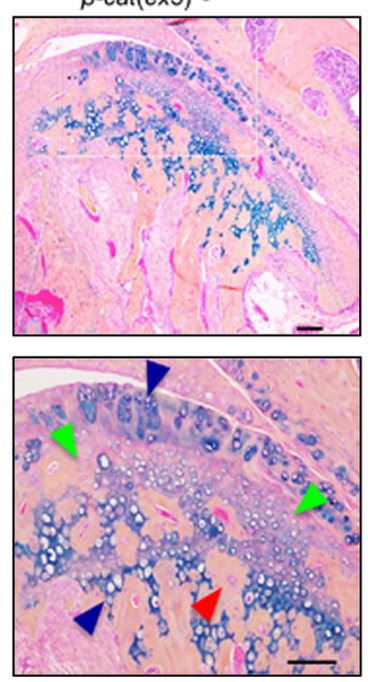

6-month-old

Scale bar $=50 \mu \mathrm{m}$

d
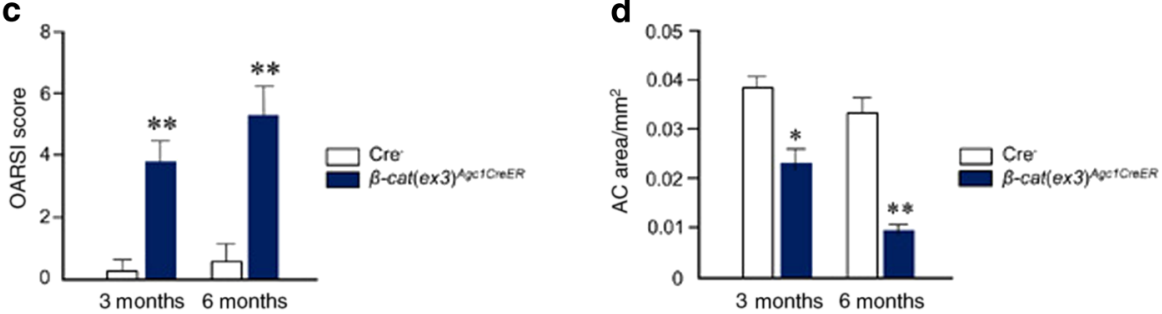

Fig. $2 \beta$-Catenin conditional activation mice $\left(\beta\right.$-cat $\left.(\text { ex3 })^{A g c 1 C r e E R}\right)$ show a progressive osteoarthritis (OA)-like phenotype in temporomandibular joint (TMJ) tissue. a TMJ samples were dissected from 3- and 6-month-old mice and Alcian blue/hematoxylin staining was performed. $\beta$-cat $(\text { ex3 })^{A g C 1 C r e E R}$ mice displayed early signs of an OA-like phenotype, including increased numbers of hypertrophic chondrocytes, the rough articular surface (blue arrowheads), loss of articular chondrocytes (green arrowheads), and new woven bone formation inside hypertrophic chondrocyte areas (red arrowheads). b Loss of cartilage tissue (green arrowheads), increased numbers of hypertrophic chondrocytes and rough articular surface (blue arrowhead), and new woven bone formation inside the hypertrophic chondrocyte areas (red arrowhead) were observed in 6-month-old $\beta$-cat $(\text { ex } 3)^{A g C 1 C r e E R}$ mice. c Analysis using the scoring system recommended by the Osteoarthritis Research Society International (OARSI) revealed cartilage destruction in 3 - and 6 -month-old $\beta$-cat $(\text { ex3) })^{A g c 1 C r e E R}$ mice $\left(* * P<0.01\right.$, versus Cre ${ }^{-}$mice; $n=5$ per group). d Histomorphometric analysis showed that TMJ cartilage areas were significantly reduced in 3-and 6-month-old $\beta$-cat(ex3) (Acr $^{\text {CreER }}$ mice ${ }^{*} P<0.05,{ }^{* *} P<0.01$, versus $C{ }^{-}$mice; values are expressed as mean \pm standard errors; $n=5$ per group)

expressing chondrocytes leads to degenerative defects resembling an OA-like phenotype in condylar cartilage. The TMJ OA is a degenerative disease with age-related joint disorder. ${ }^{25}$ Meanwhile, TMJ disorders mostly affect young women according to recent researches. $^{26,27}$ The Agc1-CreER ${ }^{T 2}$ transgenic mouse model is a valuable tool to investigate the postnatal OA development, allowing chondrocyte-specific gene targeting in an inducible manner. ${ }^{24}$ To determine the role of $\beta$-catenin in TMJ OA development in postnatal mice, we decided to induce $\beta$-catenin expression in 2 -week-old mice. $\beta$-Catenin was activated specifically in mature chondrocytes. $\beta$-cat $(\text { ex } 3)^{\text {Agc1CreER }}$ mice exhibited TMJ phenotype similar to that of human TMJ OA, including increased chondrocyte hypertrophy observed in the superficial zone of the condylar cartilage, severe loss of articular cartilage at the margins of cartilage tissue, and subchondral sclerosis. In $\beta$ catenin conditional activation mice, the accelerated catabolic effects (matrix degradation and hypertrophy) may contribute to the eventual loss of the condylar cartilage in this mouse model.

In previous studies, we demonstrated that $\beta$-cat(ex3) Co12CreER mice also showed TMJ OA-like phonotype. ${ }^{16}$ Compared to middle and deep layers, the superficial area of the condylar cartilage is relatively normal. This is probably because the Col 2 gene is not expressed in cells of the superficial layer. Col2 is mainly expressed in the middle and deep layers, ${ }^{28}$ so the condylar cartilage was not the most efficiently targeted by Co12-CreER ${ }^{T 2}$ mice., ${ }^{3,29,30}$ In the present study, we specifically determine the role of $\beta$-catenin signaling in Agc1-CreER ${ }^{T 2}$ targeting cells. Interestingly, we observed that activation of $\beta$-catenin signaling in aggrecanexpressing cells leads to dramatic damage in the superficial zone of condylar cartilage. This finding indicates that the superficial zone of the condylar cartilage could be more efficiently targeted by Agc1-CreER ${ }^{T 2}$ mice. Our findings using both transgenic mice clearly demonstrated that the proper level of $\beta$-catenin activity is critical for maintaining the integrity of the condylar cartilage in TMJ.

In this study, we also observed significant increases in the expression of collagenase (MMP13) and aggrecanases (Adamts4 and Adamts5) in $\beta$-cat(ex3) ${ }^{A g C 1 C r e E R}$ mice. In addition, there is a significant increase in the expression of ColX, the most specific

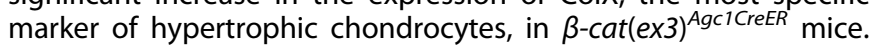
Consistent with this, increased numbers of hypertrophic chondrocytes were observed in the condylar cartilage in $\beta$-cat (ex3) ${ }^{A g c 1 C r e E R}$ mice. MMP13 and Adamts5 are the primary enzymes leading to cartilage degradation. ${ }^{31,32} \beta$-Catenin may serve as an important regulator of MMP13 and Adamts5 in hypertrophic chondrocytes. In our study, we used two different $\beta$-catenin activation mouse models to demonstrate that proper levels of $\beta$ catenin are critical in maintaining condylar cartilage integrity; 

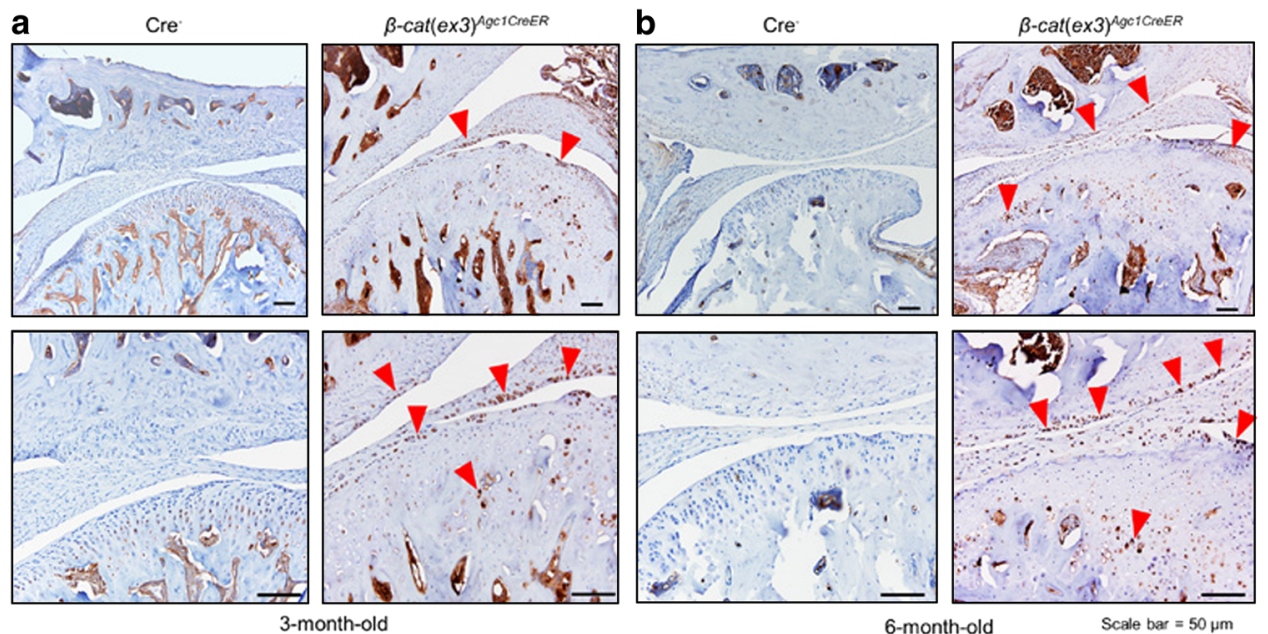

Fig. 3 Immunohistochemical (IHC) analysis of MMP13 expression. a, b MMP13 protein levels were increased in 3- and 6-month-old $\beta$-cat $(e \times 3)^{A g c i C r e E R}$ conditional activation mice, especially in the superficial zone (red arrowheads: MMP13-positive cells)

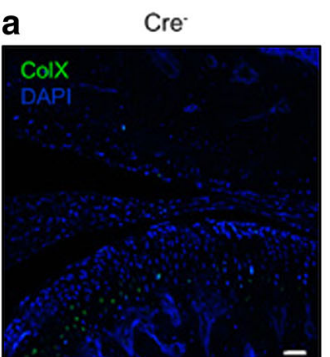

$\beta$-cat $(e \times 3)^{A B C H C r e E R}$

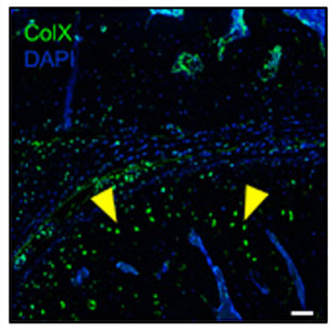

3-month-old

C

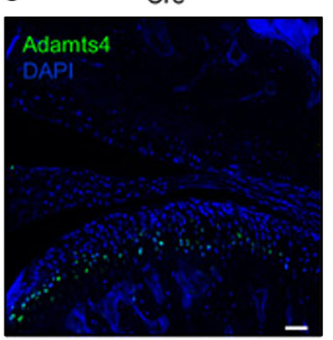

$\beta$-cat $(e \times 3)^{A g c t a r o E R}$

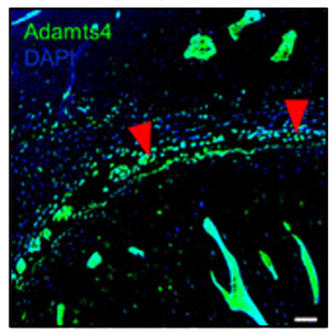

3-month-old

e

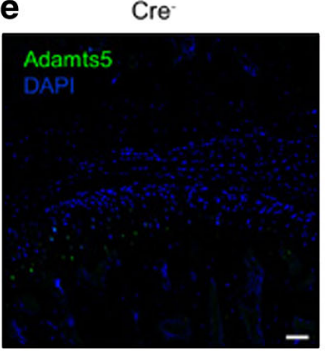

$\beta$-cat(ex3)Agc1creER

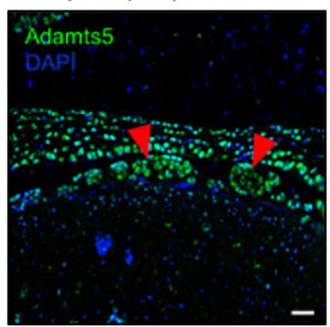

3-month-old
Cre

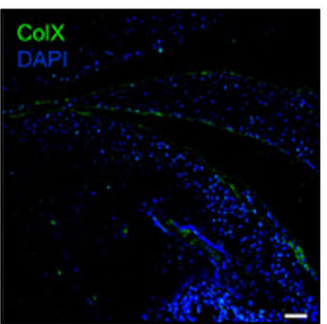

6-month-old

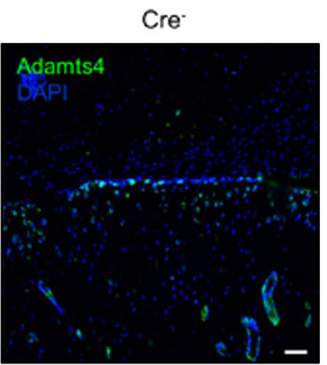

6-month-old

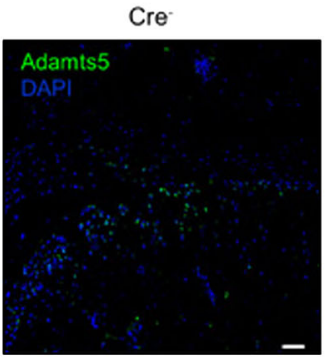

6-month-old
B-cat $(e \times 3)^{A g C}$ croes

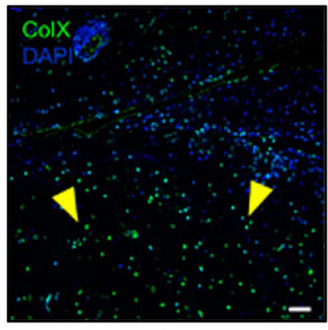

Scale bar $=20 \mu \mathrm{m}$

$\beta$-cat(ex3)Agcrcroef

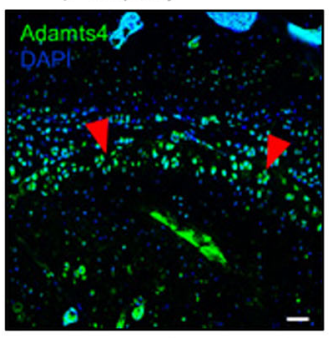

Scale bar $=20 \mu \mathrm{m}$

$\beta$-cat(ex3) ${ }^{A g C 1 C r e E R}$

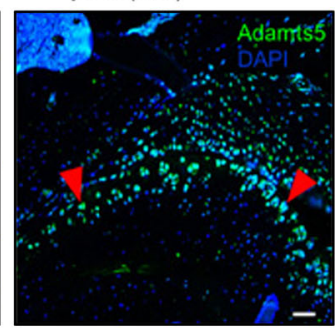

Scale bar $=20 \mu \mathrm{m}$
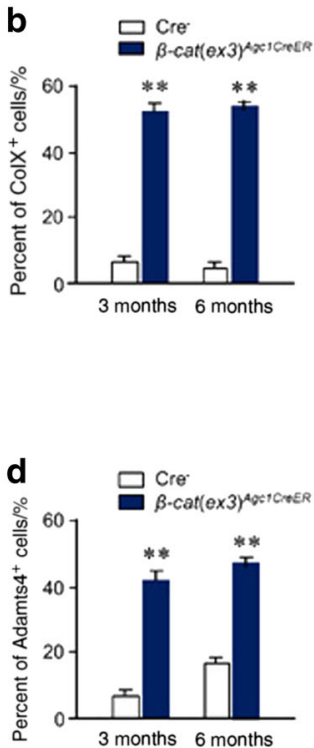

3 months 6 months

Fig. 4 Abnormal expression of the hypertrophic chondrocyte marker and matrix-degradation enzymes in 3- and 6-month-old $\beta$-cat $(\text { ex3) })^{\text {AgciCreER }}$ conditional activation mice. Immunofluorescence (IF) assays were performed to detect the expression of ColX, Adamts4, and Adamts5 proteins in $\beta$-cat $(\text { ex } 3)^{A g c 1 C r e E R}$ mice. $\mathbf{a}, \mathbf{b}$ IF results showed that Co1X expression was significantly increased in the areas that hypertrophic chondrocytes are located in $\beta$-catenin conditional activation mice. Yellow arrowheads: ColX-positive cells. c-f The expression of Adamts4 and Adamts5 was also significantly increased, especially on the surface of TMJ cartilage in $\beta$-cat $(\text { ex } 3)^{A g c 1 C r e E R}$ mice ${ }^{* *} P<0.01$; values are expressed as mean $\pm \mathrm{SE} ; n=5$ per group). Red arrowheads: Adamts4- and Adamts5-positive cells 
a
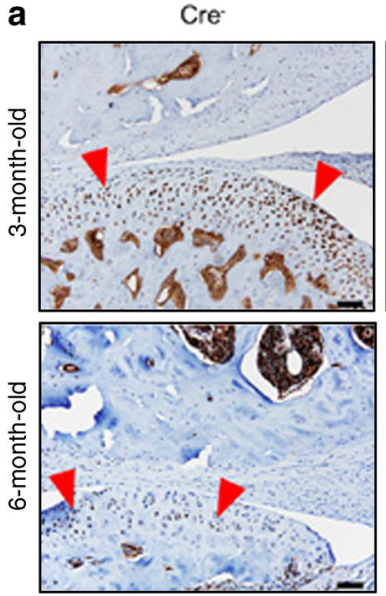

PCNA staining

d
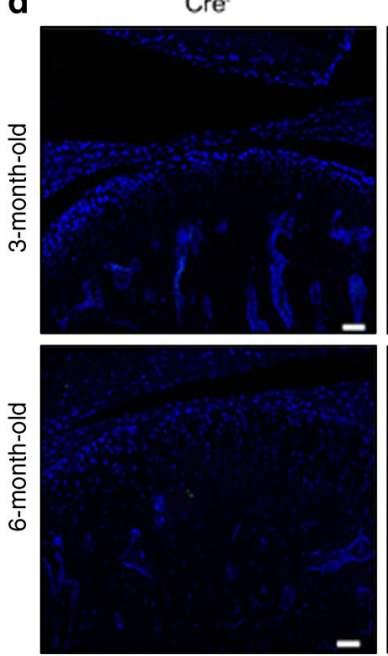

$\beta$-cat $(e \times 3)^{A g c t c r o t} R$
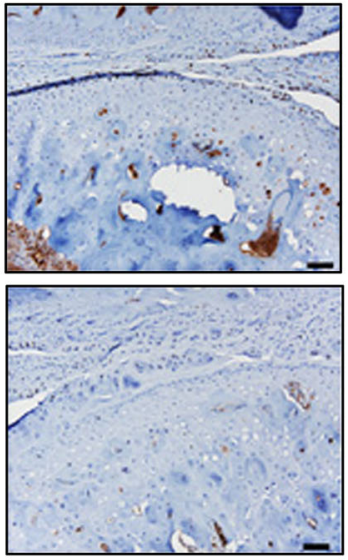

$\beta$-catt $(e \times 3) \operatorname{sectCreeR}$
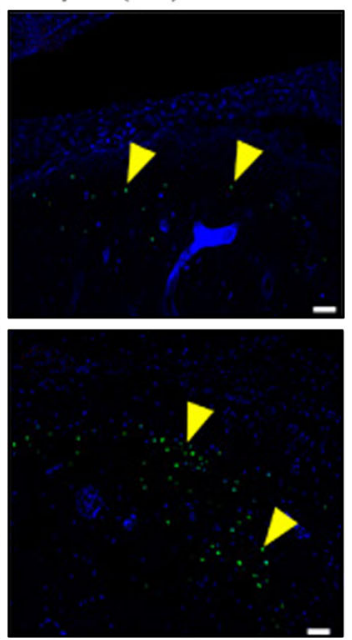

Scale bar $=20 \mu \mathrm{m}$ b

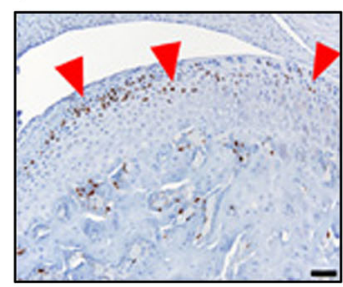

3-month-old
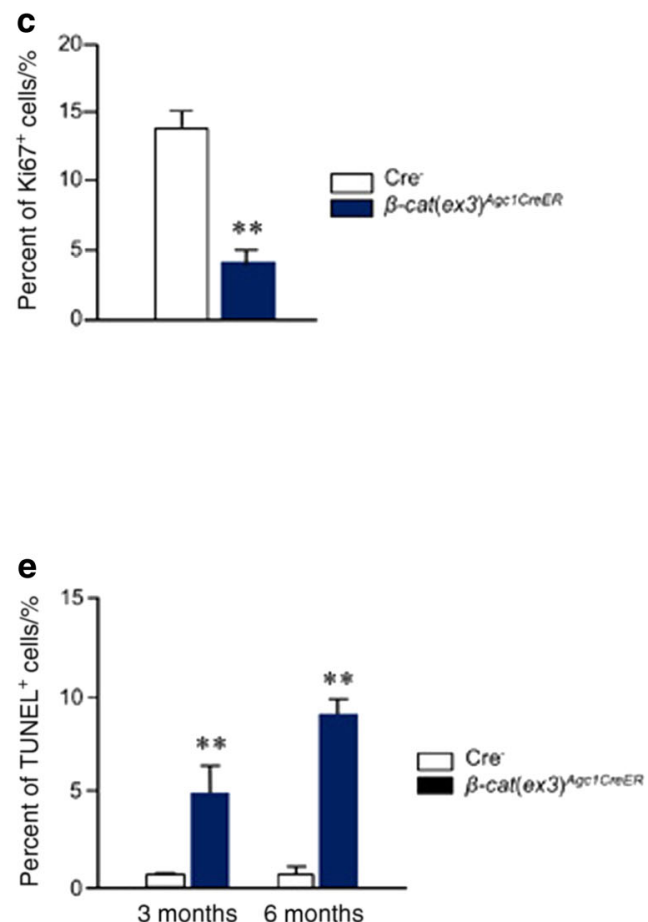

Fig. 5 Alteration of cell proliferation and apoptosis in 3- and 6-month-old $\beta$-cat $(\text { ex } 3)^{A g c 1 C r e E R}$ conditional activation mice. a Results of proliferating cell nuclear antigen (PCNA) staining revealed that cell proliferation was significantly reduced in temporomandibular joint (TMJ) chondrocytes in $\beta$-cat $(e x 3)^{A g c i C r e E R}$ mice. Red arrowheads: PCNA-positive cells. b, c Results of Ki67 staining revealed that cell proliferation, mostly in the middle layer of condylar chondrocytes, significantly reduced in TMJ cartilage in 3-month-old $\beta$-cat(ex3) $)^{A g c 1 C r e E R}$ mice. Red arrowheads: Ki67-positive cells. d, e Results of terminal deoxinucleotidyl transferasemediated dUTP-fluorescein nick end labeling (TUNEL) staining demonstrated increased numbers of apoptotic cells were detected in TMJ cartilage of $\beta$-cat $(\text { ex } 3)^{\text {AgciCreER }}$ mice $\left(* * P<0.01\right.$ versus Cre ${ }^{*}$ mice; values are expressed as mean \pm standard errors; $n=5$ per group). Yellow arrowheads: TUNEL positive cells

however, it remains unknown how $\beta$-catenin signaling is upregulated during the development of TMJ OA.

Previous reports revealed that cell proliferation and apoptosis in condylar cartilage could also be involved in $O A$ development. $^{20,33}$ The rat TMJ OA model showed histological changes, including reduced chondrocytes proliferation and increased chondrocytes apoptosis. ${ }^{34}$ It has also been reported that the OA is caused by excessive chondrocyte apoptosis. ${ }^{35}$ The superficial and/or middle zones of normal condylar cartilage have been identified as regions enriched in cells that are highly proliferative. ${ }^{19}$ In the present study, in addition to the cartilage degradation we also demonstrated decreased chondrocyte proliferation and increased chondrocyte apoptosis in $\beta$-cat $(e \times 3)^{A g c 1 C r e E R}$ mice. These changes could also contribute to the development of TMJ OA. The increased numbers of TUNEL-positive chondrocytes may reflect the enhancement of chondrocyte differentiation in the middle zone of the condylar cartilage in $\beta$-cat $(\text { ex } 3)^{A g c 1 C r e E R}$ mice. ${ }^{36}$ This is consistent with the notion that cell apoptosis of mandibular condylar could be responsible for the development and progression of TMJ OA. ${ }^{20,34}$

In summary, our study revealed that, in addition to changes in the middle and deep zones, the morphology and function of the superficial zone of cartilage could also be regulated by $\beta$-catenin signaling. We suggest that $\beta$-catenin may play important roles in chondrocyte proliferation, differentiation, and apoptosis in the condylar cartilage. Dysregulation of $\beta$-catenin signaling in chondrocytes of condylar cartilage may cause significant changes in chondrocyte function, leading to TMJ OA development. TMJ $\beta$ catenin signaling may be served as a potential therapeutic target for the development of drugs to treat TMJ OA.

\section{MATERIALS AND METHODS}

Animals

Agc1-CreER ${ }^{T 2}$ transgenic mice ${ }^{24}$ and $R O S A^{m T / m G}$ (membraneTomato/membrane-Green) reporter mice ${ }^{37}$ were obtained from Jackson Laboratories (Bar Harbor, ME, USA). $\beta$-catenin $(\text { ex3 })^{\text {flox/flox }}$ 
mice were originally reported by Harada et al. ${ }^{38}$ and we have used these mice in our previous studies. ${ }^{7,14,16} \beta$-cat(ex3) ${ }^{\text {Agc1CreER }}$ mice and the Cre-negative littermates were generated. Tamoxifen (Sigma, St. Louis, MO, USA) was administered into 2-week-old mice by intraperitoneal (i.p.) injection (1 mg per $10 \mathrm{~g}$ body weight for 5 consecutive days), $n=5$ in each group. The animal protocol of this study has been approved by the IACUC of the Rush University and all experimental methods and procedures were carried out in accordance with the approved guidelines.

Cre-recombination efficiency

$R O S A^{m T / m G}$ mice contain two loxP sites on either side of the mT cassette. Mice express red fluorescence in all cell types and tissues before Cre-recombination and green fluorescence signal can be detected after Cre-recombination. ${ }^{37}$ Agc1-CreER ${ }^{T 2}$ mice were bred with $R O S A^{m T / m G}$ mice to generate Agc1-CreER ${ }^{T 2} ; R O S A^{m T / m G}$ mice. Tamoxifen was administered into 2-week-old mice by i.p. injection ( $1 \mathrm{mg}$ per $10 \mathrm{~g}$ body weight for 5 days). Skulls were dissected after the mice were sacrificed at age 1 month, fixed in $0.2 \%$ glutaraldehyde at $4{ }^{\circ} \mathrm{C}$ for 2 days, followed by washing three times with phosphate buffered saline (PBS). Samples were decalcified in 14\% EDTA for 3 weeks, cryo-protected in $30 \%$ sucrose at $4{ }^{\circ} \mathrm{C}$ for 3 days and then embedded and processed for frozen sections. Three- $\mu$ m-thick sections were imaged with a fluorescence microscope.

Histology and histomorphometry

We dissected the skulls from $\beta$-cat $(e x 3)^{A g c 1 C r e E R}$ mice and their corresponding Cre-negative control mice. Samples were fixed in $10 \%$ neutral buffered formalin (VWR, Radnor, PA, USA) for 3 days, then decalcified with formic acid (Decal Chemical Corp., Suffern, NY, USA) for 14 days. Samples were processed and embedded in paraffin. Three- $\mu$ m-thick mid-sagittal sections at three different levels $(50 \mu \mathrm{m}$ apart) were cut from the medial compartment of the TMJ. The sections were stained with Alcian blue/hematoxylin and eosin for morphologic analysis. Three slides per mouse, five mice per group, were analyzed in the experiment. The histology data were further analyzed with OARSI scoring system as previously described. ${ }^{39,40}$ We also quantified the cartilage area using the OsteoMeasure software (OsteoMetrics, Inc., Atlanta, GA, USA).

$\mathrm{IHC}$ and IF

The paraffin sections were baked at $65^{\circ} \mathrm{C}$ overnight. Slides were then deparaffinized and rehydrated. Dako endogenous blocking reagent (S2003, Dako, Carpinteria, CA, USA) was then used to quench endogenous peroxidase for $15 \mathrm{~min}$. Non-specific binding sites were blocked with 1:10 normal horse/goat serum (S2000, Vector Laboratories, Burlingame, CA, USA) for $30 \mathrm{~min}$ at room temperature. Primary antibodies: 1:400 dilution of MMP13 (ab39012, Abcam, Cambridge, UK), 1:1 000 dilution of ColX (ab49945, Abcam, Cambridge, UK); 1:400 dilution of Admts4 (ABT178, Millipore, Billerica, MA), and 1:500 dilution of Adamts5 (ab41037 Abcam, Cambridge, UK) were added, and the slides were incubated at $4{ }^{\circ} \mathrm{C}$ overnight. For IHC assays, the secondary biotinylated goat anti-mouse antibody (BA-9200, Vector Laboratories) at the dilution of 1:200 was added for 30 min on the second day, followed by incubation with 1:250 streptavidin (21130, Pierce, Rockford, IL, USA) for $30 \mathrm{~min}$. Positive staining was detected by Romulin AEC Chromagen (Biocare Medical RAEC810L, Concord, CA, USA). For IF staining, an appropriate secondary antibody conjugated to a fluorescence probe was added, incubated at room temperature for $1 \mathrm{~h}$, rinsed in PBS, and mounted in an anti-fading mounting media (Vector Laboratories, Burlingame, CA). Results were obtained using an Olympus BX43 upright microscope (Olympus Optical, Tokyo, Japan).
Cell proliferation and apoptosis assays

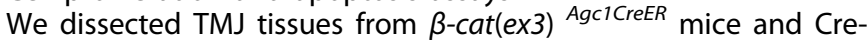
negative controls. Samples were fixed in $10 \%$ formalin, decalcified, and embedded in paraffin. The condyles were sectioned into serial sections at $3-\mu \mathrm{m}$-thick in an anterior-posterior direction. Cell proliferation was carried out using anti-PCNA and anti-Ki67 antibodies at the dilution of 1:200 (abl8197, ab16667, Abcam, Cambridge, UK) and 1:2000, respectively, as previously described. ${ }^{16}$ Apoptosis assay was carried out using a TUNEL Assay Kit according to the manufacturer's instructions (G3250, Promega, Madison, WI, USA).

Statistical analysis

The values are presented as mean \pm standard error. Statistical difference between groups was evaluated using one-way analysis of variance followed by Tukey-Kramer test and Student's $t$-test with the SPSS13.0 statistical software. ${ }^{*} P<0.05$ and ${ }^{* *} P<0.01$ are considered as significant difference between groups.

\section{ACKNOWLEDGEMENTS}

We would like to express our gratitude to Ms. Lily Yu for her help in processing and staining histological samples. This work was supported by the National Institutes of Health Grants R01 AR054465 and R01 AR070222 to D.C. This work was also partially supported by the Natural Science Foundation of China (NSFC) (grant \# 81371999 ) to D.C. T.H. was partially supported by the State Scholarship Fund (No. 201406240061), China. J.L. was partially sponsored by a grant from Shenzhen Science and Technology Innovation Committee (JCYJ20160331114205502 and JCYJ20150626090344603), and T.W. was partially supported by NSFC grants (grant \# 81301531 and 81572104), China.

\section{AUTHORS CONTRIBUTIONS}

D.C. contributed to the experimental design and data interpretation. T.H., L.Z., S.Z., Y. Z., L.L., T.W., and J.G. carried out all experiments. T.H. contributed to the manuscript preparation. D.C. and L.Y. contributed to the revision of the manuscript.

\section{ADDITIONAL INFORMATION}

Competing interest: The authors declare no competing interests.

\section{REFERENCES}

1. Slavkin, H. C. A lifetime of motion: temporomandibular joints. J. Am. Dent. Assoc. 127, 1093-1098 (1996)

2. Zhao, Y. P., Zhang, Z. Y., Wu, Y. T., Zhang, W. L. \& Ma, X. C. Investigation of the clinical and radiographic features of osteoarthrosis of the temporomandibular joints in adolescents and young adults. Oral Surg. Oral Med. Oral Pathol. Oral Radiol. Endod. 111, 27-34 (2011).

3. Chen, M., Li, S., Xie, W., Wang, B. \& Chen, D. Col2-CreER ${ }^{T 2}$, a mouse model for a chondrocyte-specific and inducible gene deletion. Eur. Cells Mater. 28, 236-245 (2014).

4. Kapila, S., Wang, W. \& Uston, K. Matrix metalloproteinase induction by relaxin causes cartilage matrix degradation in target synovial joints. Ann. NY Acad. Sci. 1160, 322-328 (2009).

5. Scrivani, S. J., Keith, D. A. \& Kaban, L. B. Temporomandibular disorders. N. Engl. J. Med. 359, 2693-2705 (2008).

6. Rando, C. \& Waldron, T. TMJ osteoarthritis: a new approach to diagnosis. Am. J. Phys. Anthropol. 148, 45-53 (2012).

7. Zhu, M. et al. Activation of beta-catenin signaling in articular chondrocytes leads to osteoarthritis-like phenotype in adult beta-catenin conditional activation mice. J. Bone Miner. Res. 24, 12-21 (2009).

8. Diarra, D. et al. Dickkopf-1 is a master regulator of joint remodeling. Nat. Med. 13 156-163 (2007).

9. Appel, $\mathrm{H}$. et al. Altered skeletal expression of sclerostin and its link to radiographic progression in ankylosing spondylitis. Arthritis Rheum. 60, 3257-3262 (2009).

10. Heiland, G. R. et al. High level of functional dickkopf-1 predicts protection from syndesmophyte formation in patients with ankylosing spondylitis. Ann. Rheum. Dis. 71, 572-574 (2012).

11. Kondo, N. et al. Intervertebral disc development is regulated by $\mathrm{Wnt} / \beta$-catenin signaling. Spine 36, 513-518 (2011). 
12. Senolt, L. et al. Low circulating Dickkopf-1 and its link with severity of spinal involvement in diffuse idiopathic skeletal hyperostosis. Ann. Rheum. Dis. 71, 71-74 (2012).

13. Xie, W., Zhou, L., Li, S., Hui, T. \& Chen, D. Wnt/ $\beta$-catenin signaling plays a key role in the development of spondyloarthritis. Ann. NY Acad. Sci. 1364, 25-31 (2016).

14. Wang, M. et al. Conditional activation of beta-catenin signaling in mice leads to severe defects in intervertebral disc tissue. Arthritis Rheum. 64, 2611-2623 (2012).

15. Kuroda, S. et al. Biomechanical and biochemical characteristics of the mandibular condylar cartilage. Osteoarthr. Cartil. 17, 1408-1415 (2009).

16. Wang, M. et al. Activation of beta-catenin signalling leads to temporomandibular joint defects. Eur. Cells Mater. 28, 223-235 (2014).

17. Singh, M. \& Detamore, M. S. Tensile properties of the mandibular condylar cartilage. J. Biomech. Eng. 130, 011009 (2008).

18. Mizoguchi, l. et al. An immunohistochemical study of regional differences in the distribution of type I and type II collagens in rat mandibular condylar cartilage. Arch. Oral Biol. 41, 863-869 (1996).

19. Hattori, S., Oxford, C. \& Reddi, A. H. Identification of superficial zone articular chondrocyte stem/progenitor cells. Biochem. Biophys. Res. Commun. 358, 99-103 (2007).

20. Chen, H., Wu, G., Sun, Q., Dong, Y. \& Zhao, H. Hyperbaric oxygen protects mandibular condylar chondrocytes from interleukin-1beta-induced apoptosis via the PI3K/AKT signaling pathway. Am. J. Transl. Res. 8, 5108-5117 (2016).

21. Loreto, C., Almeida, L. E., Trevilatto, P. \& Leonardi, R. Apoptosis in displaced temporomandibular joint disc with and without reduction: an immunohistochemical study. J. Oral Pathol. Med. 40, 103-110 (2011).

22. van der Kraan, P. M. \& van den Berg, W. B. Chondrocyte hypertrophy and osteoarthritis: role in initiation and progression of cartilage degeneration? Osteoarthr. Cartil. 20, 223-232 (2012).

23. Hanyecz, A. et al. Proteoglycan aggrecan conducting $T$ cell activation and apoptosis in a murine model of rheumatoid arthritis. Biomed. Res. Int. 2014, 942148 (2014).

24. Henry, S. P. et al. Generation of aggrecan-CreERT2 knockin mice for inducible Cre activity in adult cartilage. Genesis 47, 805-814 (2009).

25. Guarda-Nardini, L., Piccotti, F., Mogno, G., Favero, L. \& Manfredini, D. Age-related differences in temporomandibular disorder diagnoses. Cranio 30, 103-109 (2012).

26. Wieckiewicz, M. et al. Prevalence and correlation between TMD based on RDC/ TMD diagnoses, oral parafunctions and psychoemotional stress in Polish university students. Biomed. Res. Int. 2014, 472346 (2014).

27. Kogawa, E. M., Calderon, P. D., Lauris, J. R., Pegoraro, L. F. \& Conti, P. C. Evaluation of minimum interdental threshold ability in dentate female temporomandibular disorder patients. J. Oral Rehabil. 37, 322-328 (2010).

28. Hirschmann, P. N. \& Shuttleworth, C. A. The collagen composition of the mandibular joint of the foetal calf. Arch. Oral Biol. 21, 771-773 (1976).
29. Chen, M. et al. Generation of a transgenic mouse model with chondrocytespecific and tamoxifen-inducible expression of Cre recombinase. Genesis 45, 44-50 (2007).

30. Zhu, M., Chen, M., Lichtler, A. C., O'Keefe, R. J. \& Chen, D. Tamoxifen-inducible Crerecombination in articular chondrocytes of adult Col2a1-CreER(T2) transgenic mice. Osteoarthr. Cartil. 16, 129-130 (2008).

31. Neuhold, L. A. et al. Postnatal expression in hyaline cartilage of constitutively active human collagenase-3 (MMP-13) induces osteoarthritis in mice. J. Clin. Invest. 107, 35-44 (2001).

32. Glasson, S. S. et al. Deletion of active ADAMTS5 prevents cartilage degradation in a murine model of osteoarthritis. Nature 434, 644-648 (2005).

33. Yasuhara, R. et al. Roles of beta-catenin signaling in phenotypic expression and proliferation of articular cartilage superficial zone cells. Lab. Invest. 91, 1739-1752 (2011).

34. Jiao, K. et al. Death and proliferation of chondrocytes in the degraded mandibular condylar cartilage of rats induced by experimentally created disordered occlusion. Apoptosis 14, 22-30 (2009).

35. Kim, H. A. \& Blanco, F. J. Cell death and apoptosis in osteoarthritic cartilage. Curr. Drug Targets 8, 333-345 (2007).

36. Jing, J. et al. Osterix couples chondrogenesis and osteogenesis in post-natal condylar growth. J. Dent. Res. 93, 1014-1021 (2014).

37. Muzumdar, M. D., Tasic, B., Miyamichi, K., Li, L. \& Luo, L. A global doublefluorescent Cre reporter mouse. Genesis 45, 593-605 (2007).

38. Harada, N. et al. Intestinal polyposis in mice with a dominant stable mutation of the beta-catenin gene. EMBO J. 18, 5931-5942 (1999).

39. Glasson, S. S., Chambers, M. G., Van Den Berg, W. B. \& Little, C. B. The OARSI histopathology initiative recommendations for histological assessments of osteoarthritis in the mouse. Osteoarthr. Cartil. 18, 17-23 (2010).

40. Shen, J. et al. Deletion of the transforming growth factor beta receptor type II gene in articular chondrocytes leads to a progressive osteoarthritis-like phenotype in mice. Arthritis Rheum. 65, 3107-3119 (2013).

(c) (i)

Open Access This article is licensed under a Creative Commons Attribution 4.0 International License, which permits use, sharing, adaptation, distribution and reproduction in any medium or format, as long as you give appropriate credit to the original author(s) and the source, provide a link to the Creative Commons license, and indicate if changes were made. The images or other third party material in this article are included in the article's Creative Commons license, unless indicated otherwise in a credit line to the material. If material is not included in the article's Creative Commons license and your intended use is not permitted by statutory regulation or exceeds the permitted use, you will need to obtain permission directly from the copyright holder. To view a copy of this license, visit http://creativecommons. org/licenses/by/4.0/.

(c) The Author(s) 2018 